\title{
Beran, Fabian; Nuissl, Henning (2019): Verdrängung auf angespannten Wohnungsmärkten. Das Beispiel Berlin.
}

\author{
Christian Diller \\ Eingegangen: 9. Oktober 2020 - Angenommen: 2. November 2020 - Online veröffentlicht: 4. Januar 2021
}

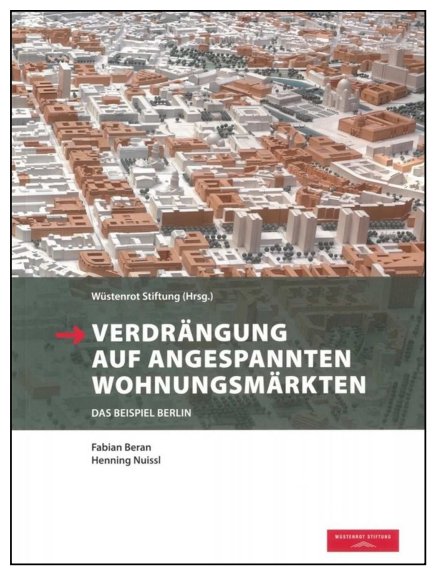

In den letzten Jahren erlebte Deutschland wie viele andere europäische Länder einen Reurbanisierungsschub. Dieser betraf vor allem die Millionenstädte, aber zunehmend auch Großstädte ab 500.000 Einwohnern. Ob die Wohnungsfrage in Deutschland eine neue soziale Frage ist, mag kontrovers gesehen werden (vgl. Sagner/Stockhausen/Voigtländer 2020); ein gravierender Wohnungsmangel ist zumindest in einer Reihe von Großstädten nicht zu bestreiten. Dort haben viele innerstädtische Quartiere zum Teil öffentliche, zum Teil marktinduzierte bauliche und funktionale Aufwertungen erfahren und es stellt sich die Frage nach deren sozialräumlichen Auswirkungen. Entsprechend erlebte die wissenschaftliche und gesellschaftliche Debatte zu gentrifica-

Prof. Dr. Christian Diller, Institut für Geographie, Professur für Raumplanung und Stadtgeographie, Justus-Liebig-Universität Gießen, Senckenbergstraße 1, 35390 Gießen christian.diller@geogr.uni-giessen.de

\section{(c) (1) (2)} article is published under the Creative Commons tion in Deutschland eine große Renaissance. Dies resultiert vor allem aus dem auch mit der Gentrifizierung möglicherweise verbundenen Phänomen der Verdrängung. Allerdings ist es der Gentrifizierungsforschung, auch wenn sie sich explizit dem Aspekt der Verdrängung widmete (vgl. Helbrecht 2016), bislang noch nicht wirklich überzeugend geglückt, den Zusammenhang zwischen quartierbezogenen Aufwertungen und Verdrängungen (in einem ausreichend differenzierten Begriffsverständnis) empirisch schlüssig nachzuweisen, sprich: vor allem auch belastbare Abschätzungen von Verdrängungsraten in Quartieren vorzulegen.

In dem von Fabian Beran und Henning Nuissl (beide am Geographischen Institut der Humboldt-Universität zu Berlin tätig) erarbeiteten und von der Wüstenrot-Stiftung herausgegebenen Buch geht es um den Aspekt, der zu den am kontroversesten diskutierten in der Wohnungs- und Quartiersentwicklungspolitik gehört: die Frage der Verdrängung aus Mietwohnungen und ihre Implikationen für Wohnquartiere. Die erste Auffälligkeit des von den Autoren durchgeführten Forschungsprojekts, auf dem das Buch basiert, ist, dass das Wort ,gentrification“ weder im Titel noch in den Kapitelüberschriften auftaucht. Damit steht die Studie von ihrem Zugang her zunächst gar nicht in der in Deutschland in den 1980er-Jahren aufkommenden Gentrifizierungsforschung, sondern knüpft eher an Untersuchungen an, die seit den späten 1970er-Jahren die Folgen der Stadterneuerungspolitik betrachteten, von denen Verdrängung eine war (z.B. Becker/Schulz zur Wiesch 1982). Gleichwohl kann bei der umfassenden und sehr gelungenen Darstellung des Standes der Verdrängungsforschung im ersten Abschnitt des Buches (Kapitel 1-4) der Bezug zur Gentrifizierungsdebatte gar nicht vermieden werden, da diese in ihrer Entwicklung viele Beiträge geliefert hat, um die Ursachen möglicher aufwertungsbedingter Verdrängung zu analysieren.

Im zweiten Teil des Buches wird dann in Kapitel 5 der Entwicklung auf dem Berliner Wohnungsmarkt und ersten 
Hinweisen auf Verdrängung auf der Basis der verfügbaren Daten zu Demographie, Wohnungsangebot, Mieten und weiteren nachgegangen. Hervorzuheben ist sodann der in Kapitel 6 von Guido Schulz vorgestellte Ansatz, in dem aufwertungsbedingte Verdrängungswahrscheinlichkeiten auf der Nachbarschaftsebene flächendeckend für die Stadt ermittelt werden, indem die Mietenentwicklung und die Fortzugsraten in Relation gesetzt werden. Im Ergebnis zeigt sich für den Zeitraum 2010 bis 2018 für ganz Berlin eine geschätzte Verdrängungsrate (Anteil der Wegzüge, die statistisch durch Mietsteigerungen zu erklären sind) von $4 \%$, die in einzelnen innerstädtischen Quartieren $8 \%$ übersteigt. Dieser Wert zeigt wohlgemerkt nicht tatsächliche Verdrängung in all ihren Facetten an, die ja letztlich nur auf der Individualebene beurteilt werden kann, aber er ist ein belastbarer Hinweis auf aufwertungsbedingte statistische Verdrängungswahrscheinlichkeiten. Eine weitere Facette wird dem Band in Kapitel 7 hinzugefügt, in dem die Behandlung des Themas „Verdrängung“ in der Berliner Presse behandelt wird.

Den Hauptteil des Bandes (Kapitel 8-15) bildet die Berliner Verdrängungsstudie, basierend auf der Promotionsarbeit von Fabian Beran an der Humboldt-Universität zu Berlin. Hier erfolgt zunächst eine systematische und intensive Auseinandersetzung mit dem Begriff der Verdrängung. Maßgeblich sind hier die Definitionen von Grier und Grier (1980), die das Moment der Umstände des Haushalts beim Wegzug betonen, und von Marcuse (1985), die vier unterschiedliche Formen der Verdrängung unterscheiden: direkte physische und ökonomische Verdrängung, Verdrängungsketten, ausschließende Verdrängung und Verdrängungsdruck. Auch wenn Marcuse mit dieser Unterscheidung seinerzeit wertvolle Pionierarbeit geleistet hatte, die z.B. in Überlegungen von Holm (2012) aufgenommen wurde, hat gerade auch die kritische Stadtforschung diese wichtige Differenzierung von Verdrängungstypen in ihren Arbeiten und Diskussionsbeiträgen zu selten sauber durchgehalten. Häufig wurde allerdings für deutsche Städte im Rahmen von quantitativen Gentrifizierungsanalysen lediglich der Aspekt des Exclusionary Displacement, der ausschließenden Verdrängung herausgearbeitet. Damit ist folgendes Phänomen angesprochen: Eine Mietpartei zieht aus einer Wohnung - aus welchen Gründen auch immer, also auch aus freiwilligem Anlass - aus und für die neue Mietpartei wird danach die Miete so stark erhöht, dass die alte Mietpartei sich diese nunmehr nicht mehr leisten könnte, wenn sie dort wieder wohnen wollen würde. Bezogen auf eine Gesamtstadt bedeutet dies, dass die Wahlmöglichkeiten für eine neue Wohnung für bestimmte Sozialstatusgruppen drastisch eingeschränkt werden. Dieser Prozess ist in vielen deutschen Großstädten nachweisbar und bedarf der gesellschaftspolitischen Problematisierung. Aber es ist vor allem ein Prozess der strukturellen Exklusion, der leider in Diskursen nicht selten mit der Form der direkten Verdrängung vermischt wird.

Durch ihre differenzierte Herangehensweise an den Verdrängungsbegriff entgehen Fabian Beran und Henning Nuissl jedoch dieser Gefahr. Nachdem in Kapitel 8 die theoretischen Überlegungen zum Prozess der Verdrängung auf der Individualebene dargestellt wurden, wird in Kapitel 9 das methodische Design der empirischen Analyse vorgestellt: eine Befragung von zwischen 2013 und 2015 aus den Bezirken Friedrichshain-Kreuzberg und Mitte Weggezogenen.

In der Befragung wurden - und das ist das methodische Hauptverdienst der Arbeit - die unterschiedlichen Formen von Verdrängung operationalisiert. Die Grundgesamtheit aller in dem Zeitraum aus dem Untersuchungsgebiet Weggezogenen lag bei gut 100.000 Personen, daraus wurde eine Zufallsstichprobe von 10.000 gezogen, an die ein Fragebogen versandt wurde. Der Rücklauf von 2082 auswertbaren Bögen kann als sehr gut eingeschätzt werden. Er lag deutlich über dem Wert aus anderen ambitionierten Untersuchungen zu der Fragestellung (z. B. Friedrichs/Blasius 2016). Die Überprüfung ergab zwar, dass Frauen und deutsche Staatsangehörige in der Stichprobe gemessen an ihrem Bevölkerungsanteil überrepräsentiert sind, dennoch kann die Validität der Befragung als sehr gut eingeschätzt werden.

In der mehrstufigen Auswertung der Befragung werden dann die Ursachen der Wegzüge aus dem Untersuchungsgebiet genauer und stufenweise differenziert analysiert. Maßgeblich ist dabei vor allem die Unterscheidung zwischen direkter und kultureller Verdrängung, die intensiv anhand der Literatur diskutiert wird. Nach den Ergebnissen der Studie wurden - durch baulichen Verfall oder Aufwertung, direkten Druck der Vermieter, Verkauf, Kündigung, Mieterhöhung - 313 der 2082 Befragten, also $15 \%$, direkt verdrängt. Dazu kommen weitere $8 \%$, für die aufgrund der Fragen zu ihrer Einschätzung der Veränderungen im Gebiet auf kulturelle Verdrängung geschlossen werden kann.

Diese zunächst sehr hoch erscheinende Zahl relativiert sich allerdings etwas durch die vertieften Betrachtungen: In Kapitel 11 werden die Verdrängungsgründe und die Verdrängten differenzierter analysiert. Besondere Aufmerksamkeit gilt dabei der Sequenzialität des Verdrängungsprozesses. Wie die meisten Mobilitätsentscheidungen ist ein Wegzug meist durch mehrere Faktoren bedingt, deren Bedeutung im Entscheidungsprozess auch variieren kann. In Kapitel 12 wird der Prozess der Wahl des neuen Wohnstandortes beleuchtet und es werden die Zuzugsorte der aus den beiden Bezirken Weggezogenen ermittelt. Im Ergebnis werden die Befunde der Wanderungsforschung bestätigt, wonach neue Wohnungen möglichst in der Nähe der alten Wohnungen gesucht werden. Darüber hinaus ist 
die Erkenntnis markant, dass die Verdrängten sogar noch eher in die Nähe des alten Wohnorts, möglichst dasselbe Quartier, ziehen wollen und dies auch weitgehender tun als die Nicht-Verdrängten.

In Kapitel 13 wird die Wohnsituation der Befragten nach dem Wegzug betrachtet. Bemerkenswert ist dabei vor allem, dass die Verdrängten ihre Wohnsituation vor dem Umzug in einer Reihe von Aspekten deutlich schlechter empfanden als die freiwillig Umgezogenen, dass jedoch die beiden Gruppen in der Einschätzung der Situation nach dem Umzug nur noch wenig auseinanderliegen. In Kapitel 14 wird der Thematik der kulturellen Verdrängung nachgegangen, indem der Anteil der kulturell Verdrängten kleinräumig dargestellt wird. Hier bestätigt sich zwar die Vermutung besonders hoher Raten an einigen zentralen Hotspots der Touristifizierung. Jedoch fallen auch andere Gebiete in peripheren Lagen auf, für die das Phänomen der kulturellen Überfremdung andere Ursachen haben muss.

Im zusammenfassenden Kapitel 15 betonen die Autoren nochmals die Sinnhaftigkeit einer klaren analytischen Trennung der Begriffe Verdrängung und Gentrifizierung. Beides sind Prozesse, die sich räumlich überlagern können und auch kausal miteinander verbunden sein können, aber nicht sein müssen. Insofern macht auch diese Studie die Problematik der Definitionen von ,gentrification“ deutlich, die in der Tradition von Marcuse (1985) Verdrängung als konstitutives Wesensmerkmal von Gentrifizierung ansehen. Die Studie belegt zwar, dass Verdrängung aus einer Wohnung auch in einem hochentwickelten Sozialstaat wie Deutschland zumindest in einigen Bereichen in Großstädten ein quantitativ relevantes Phänomen ist. Welche wohnungspolitischen Instrumente daher wie zu entwickeln wären, wird von den Autoren im Schlusskapitel angedeutet. Die Studie belegt jedoch gleichzeitig, dass Verdrängungen komplexe Prozesse sind, die durch die gentrification in den Wohngebieten der Verdrängten nur zum Teil bedingt sind.

Insgesamt kann der Band ohne Übertreibung als eine der der wichtigsten theoretisch fundierten empirischen Untersuchungen der deutschen Stadtforschung der letzten Jahre angesehen werden. Sein Verdienst ist es zunächst, die Problematik der Verdrängung aus Wohnungen in einer bislang nicht gelungenen Differenziertheit zu strukturieren und empirisch zu fundieren. Die klar und nachvollziehbar berechnete Zahl von über $20 \%$ direkt und kulturell Verdrängter aus den Gebieten ist eine der wenigen (ein älteres Beispiel: Blasius 1993) gebietsbezogenen Verdrängungsquoten, die für Quartiere in einer deutschen Stadt ermittelt wurden. Damit kann zum einen die politische Brisanz des Themas verdeutlicht, zum anderen aber auch die Debatte dazu zu versachlicht werden.

Über diesen kaum zu überschätzenden Beitrag zur Verdrängungsforschung hinaus dürfte die Studie aber auch ent- scheidende Impulse für die Gentrifizierungsforschung geben. Auf theoretischer Ebene ist den Autoren in dem Band das Aufzeigen der Schnittmengen von Verdrängungs- und Gentrifizierungsforschung nahezu vollständig gelungen. In den empirischen Arbeiten fehlt in dem Band für die Verbindung der beiden Forschungsstränge lediglich das ,I-Tüpfelchen': nämlich die räumliche Überlagerung der quartiersbezogenen Gentrifizierungs-/Verdrängungsverdachtsanalysen von Guido Schulz (Kapitel 6) und der Wegzugsanalysen von Fabian Beran (Kapitel 8-15). Aufgrund der unterschiedlichen Betrachtungszeiträume der beiden Auswertungen war dies im Rahmen dieser Untersuchung nicht seriös möglich. Methodisch wurde mit dem Band jedoch ein klarer Weg gezeigt, wie mit hohem aber dennoch leistbarem Aufwand die die „Gentrification“-Debatte beherrschende Verdrängungsthematik auch für die quantitativ orientierte Stadtforschung besser in den Griff zu bekommen ist.

Ein theoretisch überaus anregendes und auf in beispielhafter Seriösität durchgeführter empirischer Analyse beruhendes Buch. Chapeau!

\section{Vollständige bibliographische Angaben des rezensierten Werkes:}

Beran, F.; Nuissl, H. (2019): Verdrängung auf angespannten Wohnungsmärkten. Das Beispiel Berlin. Ludwigsburg: Verlag Wüstenrot-Stiftung. 240 Seiten, 62 Abbildungen, 13 Tabellen, 9 Anhangtabellen.

\section{Literatur}

Becker, H.; Schulz zur Wiesch, J. (Hrsg.) (1982): Sanierungsfolgen. Eine Wirkungsanalyse von Sanierungsmaßnahmen in Berlin. Stuttgart. $=$ Schriften des Deutschen Instituts für Urbanistik 70.

Blasius, J. (1993): Gentrification und Lebensstile. Eine empirische Untersuchung. Wiesbaden.

Friedrichs, J.; Blasius, J. (Hrsg.) (2016): Gentrifizierung in Köln. Soziale, ökonomische, funktionale und symbolische Aufwertungen. Opladen.

Grier, G.; Grier, E. (1980): Urban Displacement: A Renaissance. In: Laska, S.B.; Spain, D. (Hrsg): Back to the City. Issues in Neighborhood Renovation. New York, 252-268.

Helbrecht, I. (Hrsg.) (2016): Gentrifizierung in Berlin. Verdrängungsprozesse und Bleibestrategien. Bielefeld.

Holm, A. (2012): Gentrification. In: Eckardt, F. (Hrsg.): Handbuch Stadtsoziologie. Wiesbaden, 661-687. https:// doi.org/10.1007/978-3-531-94112-7_29

Marcuse, P. (1985): Gentrification, Abandonment, and Displacement. Connections, Causes and Policy Responses in New York City. In: Journal of Urban and Contemporary Law 28, 1, 195-240. 
Sagner, P.; Stockhausen, M.; Voigtländer, M. (2020): Wohnen - die neue soziale Frage? Köln. = IW-Analysen 136. 